\title{
Heart and Skeletal Muscles: Linked by Autonomic Nervous System
}

\author{
Claudio Gil Araujo ${ }^{10}$ and Jari Antero Laukkanen ${ }^{(0)}$ \\ Exercise Medicine Clinic - CLINIMEX, ${ }^{1}$ Rio de Janeiro, RJ - Brazil \\ University of Jyvaskyla - Faculty of Sport and Health Sciences, ${ }^{2}$ Jyvaskyla - Finland \\ Short Editorial related to the article: Sympatho-Vagal Imbalance is Associated with Sarcopenia in Male Patients with Heart Failure
}

The autonomic nervous system (ANS) plays a fundamental role in maintaining cell homeostasis and human life. The functionality of the heart and skeletal muscles is partially modulated by sympathetic and parasympathetic branches of the ANS both in resting and exercising conditions.

Substantial literature attests to the fact that objective indices of muscle strength reflect health status. It is well-established that heart failure is typically accompanied by skeletal muscle abnormalities that contributed to the exercise intolerance and poor health-related quality of life usually seen in these patients. ${ }^{1,2}$ Indeed, while a decrease in muscle mass and strength is naturally seen with ageing, especially after the fifth decade of life, this is especially more relevant in middle-aged and older patients with heart failure.

Several decades ago, the term sarcopenia was proposed as a medical expression to describe the universal and involuntary loss in muscle mass that occurs with age. ${ }^{3}$ However, despite the fact that several criteria were proposed to characterize it, a growing body of knowledge understanding its pathophysiology and confirming its clinical and epidemiological relevance and being listed in ICD-10, ${ }^{4}$ still today, it remains rarely evaluated in daily medical practice.

In a Brazilian and German collaborative research study, Fonseca et al. ${ }^{5}$ analyzed data from 116 male patients with heart failure with reduced ejection fraction (HFrEF) that were submitted to a maximal cycling cardiopulmonary exercise testing using a ramp protocol. In addition, using a microneurography technique, muscle sympathetic nerve activity was directly recorded from the peroneal nerve and parasympathetic activity was estimated by the magnitude of heart rate decay in the first two minutes after the maximal cycling exercise test. Measurements were obtained from dual-energy X-ray absorptiometry (DEXA) and handgrip strength to reflect, respectively, body composition and

\section{Keywords}

Heart Failure; Myocardium; Muscle,Skeletal; Autonomic Nervous System; Homeostasis; Sarcopenia; Sympathetic Nervous System.

Mailing Address: Claudio Gil Araujo •

Rua Siqueira Campos, 93/101-103, Postal Code 22031-072, Rio de Janeiro, RJ - Brazil

E-mail: cgaraujo@iis.com.br

DOI: $10.5935 / a b c .20190097$ muscle strength. Based on these measurements and applying standard literature criteria, the authors were able to identify the presence or absence of sarcopenia in their set of patients. We acknowledge the authors by their study originality that could add a significant contribution to the existing body of knowledge in the research area.

Combining all these data, they searched for a link between heart-skeletal muscle abnormalities and ANS dysfunction and tried to quantify the association between sympathetic ANS abnormalities and sarcopenia in male patients with clinically stable heart failure. They have identified sarcopenia in 33 (28\%) of HFrEF patients and their results indicated that these patients had significantly distinct results to the ANS variables assessed when compared as to the group of patients without sarcopenia. ${ }^{5}$ Moreover, they found a significant although quite modest correlation $(r=-0.29)$ between appendicular muscle mass and muscle sympathetic nerve activity. Looking in more details their results, it is possible to note that there is a considerable overlapping between the results of HFrEF patients with and without sarcopenia, which may diminish the clinical value.

Based on their current study by Fonseca et al., ${ }^{5}$ we can speculate that if they have used other assessment methods, like the 4-second exercise test, ${ }^{6,7}$ - a very specific one for assessing cardiac vagal activity - and handgrip strength relative to body weight or maximal muscle power ${ }^{8}$ or even a simple functional test like the sitting-rising test, ${ }^{9}$ all of them, more specific to assess dynapenia, a likely clinically more relevant issue than sarcopenia, ${ }^{10,11}$ they could have found additional discriminative values.

Finally, perhaps regular exercise/physical exercise could be the way to improve the health status of HFpEF patients. In acknowledging that this study showed an association between cardiac ANS dysfunction and heart and skeletal muscle abnormalities and knowing that regular aerobic and resistance exercise improves cardiac ANS modulation, including reducing the risk of ventricular fibrillation on the occurrence of a myocardial infarction, ${ }^{12}$ and are strongly recommended as part of the medical treatment of patients with sarcopenia and for those with $\mathrm{HFpEF}{ }^{13}$ it is quite motivating to think about an expected next step in research: a randomized controlled trial with exercise training intervention. Such study would assess if the ANS dysfunction reported by Fonseca et al. ${ }^{5}$ is subject to reversal, and if so, how this would improve quality of life and other major health outcomes in patients with HFpEF. 


\section{References}

1. Engel PJ. Effort intolerance in chronic heart failure: what are we treating? J Am Coll Cardiol. 1990;15(5):995-8.

2. Pugliese NR, Fabiani I, Santini C, Rovai I, Pedrinelli R, Natali A, et al. Value of combined cardiopulmonary and echocardiography stress test to characterize the haemodynamic and metabolic responses of patients with heart failure and mid-range ejection fraction. Eur Heart J Cardiovasc Imaging. Feb 11 2019. [Epub ahead of print]. doi: 10.1093/ehjci/jez014

3. Evans WJ, Campbell WW. Sarcopenia and age-related changes in body composition and functional capacity. J Nutr. 1993;123(2 Suppl):465-8.

4. Anker SD, Morley JE, von Haehling S. Welcome to the ICD-10 code for sarcopenia. J Cachexia Sarcopenia Muscle. 2016;7(5):512-4.

5. Fonseca G, Santos MRD, Souza FR, Costa M, Haehling SV, Takayama L, et al. Sympatho-vagal imbalance is associated with sarcopenia in male patients with heart failure. Arq Bras Cardiol. 2019; 112(6):739-746.

6. Araujo CGS, Castro CLB, Nobrega ACL. Heart rate responses to deep breathing and 4-seconds of exercise before and after pharmacological blockade with atropine and propranolol. Clin Auton Res. 1992;2(1):35-40.

7. Araújo CG, Castro CL, Franca JF, Ramos PS. 4-Second exercise test reference values for ages 18-81 years. Arq Bras Cardiol. 2015;104(5):366-74.
8. Araujo CG, Castro CL, Franca JF, Laukkanen JA, Hamar D, Myers J. Muscle power in upright row movement: predictor of all-cause mortality in individuals between 41 and 85 years of age: preliminary results. Eur J Prev Cardiol. 2019;26(6 (suppl)).

9. Brito LBB, Ricardo DR, Araújo DSMS, Ramos PS, Myers J, Araújo CGS. Ability to sit and rise from the floor as a predictor of all-cause mortality. Eur J Prev Cardiol. 2014;21(7):892-8.

10. Mitchell WK, Williams J, Atherton P, Larvin M, Lund J, Narici M Sarcopenia, dynapenia, and the impact of advancing age on human skeletal muscle size and strength; a quantitative review. Front Physiol. 2012;3:260.

11. Clark BC, Manini TM. Sarcopenia $=/=$ dynapenia. $J$ Gerontol A Biol Sci Med Sci. 2008;63(8):829-34.

12. De Ferrari GM, Dusi V, Ruffinazzi M, Gionti V, Cacciavillani L, Noussan P, et al. Physical inactivity is a risk factor for primary ventricular fibrillation. J Am Coll Cardiol. 2019;73(16):2117-8.

13. Aagaard P, Suetta C, Caserotti P, Magnusson SP, Kjaer M. Role of the nervous system in sarcopenia and muscle atrophy with aging: strength training as a countermeasure. Scand J Med Sci Sports. 2010;20(1):49-64 